\title{
GENERALIZED CHEBYSHEV POLYNOMIALS ASSOCIATED WITH AFFINE WEYL GROUPS
}

\author{
MICHAEL E. HOFFMAN AND WILLIAM DOUGLAS WITHERS
}

\begin{abstract}
We begin with a compact figure that can be folded into smaller replicas of itself, such as the interval or equilateral triangle. Such figures are in one-to-one correspondence with affine Weyl groups. For each such figure in $n$-dimensional Euclidean space, we construct a sequence of polynomials $P_{k}: \mathbf{R}^{n} \rightarrow \mathbf{R}^{n}$ so that the mapping $P_{k}$ is conjugate to stretching the figure by a factor $k$ and folding it back onto itself. If $n=1$ and the figure is the interval, this construction yields the Chebyshev polynomials (up to conjugation). The polynomials $P_{k}$ are orthogonal with respect to a suitable measure and can be extended in a natural way to a complete set of orthogonal polynomials.
\end{abstract}

1. Introduction. The Chebyshev polynomials $T_{k}$, by virtue of the definition

$$
T_{k}(\cos x)=\cos k x,
$$

can be thought of as arising from the stretching and folding of the interval $[0, \pi]$. More precisely, the function $\cos ^{-1} \circ T_{k} \circ \cos$ stretches the interval by a factor of $k$ and returns it to $[0, \pi]$ by folds at integer multiples of $\pi$. It follows immediately from (1) that

$$
T_{k} \circ T_{l}=T_{k l}
$$

for any positive integers $k$ and $l$. Property (2) is rather unusual for a sequence of polynomials; up to conjugation, the only sequences of polynomials in one variable that have it are the Chebyshev polynomials and the power functions $x^{k}[\mathbf{1 1}$, Chapter 4].

Suppose now we have a geometric figure in $\mathbf{R}^{n}$ that, like the interval, can be folded (i.e., mapped by reflections in hyperplanes) into smaller replicas of itself. We call such a figure foldable. As is shown in $\S 2$, such figures are convex polytopes and are in one-to-one correspondence with the groups of reflections they generate. In $\S 3$, the well-known theory of such groups is reviewed: they are affine Weyl groups of root systems, and the classification of the latter is classical.

In $\S 4$ we define, for any foldable figure $F$, a function $h: \mathbf{R}^{n} \rightarrow \mathbf{R}^{n}$ which we call the generalized cosine associated with $F$. This function has two properties, which generalize those of the cosine in the classical case: it is invariant under the group of reflections associated with $F$, and $h(k \mathbf{x})$ is a polynomial in $h(\mathbf{x})$ for

Received by the editors October 29, 1986.

1980 Mathematics Subject Classification (1985 Revision). Primary 33A65; Secondary 33A75, $51 \mathrm{~F} 15$.

Key words and phrases. Chebyshev polynomials, affine Weyl groups, orthogonal polynomials, reflection groups, root systems.

Both authors were partially supported by grants from the Naval Academy Research Council. 
any positive integer $k$. We can then define generalized Chebyshev polynomials (or folding polynomials) associated with $F$ by

$$
P_{k}(h(\mathbf{x}))=h(k \mathbf{x})
$$

for integer $k \geq 1, \mathbf{x} \in \mathbf{R}^{n}$. As with the Chebyshev polynomials, the function $h^{-1} \circ P_{k} \circ h$ stretches $F$ by a factor of $k$ and returns to $F$ by folding.

If $F$ is the interval, the polynomials $P_{k}$ are conjugate to the classical Chebyshev polynomials (see $\S 7$ ). As another example, let $F$ be the equilateral triangle. The associated group of reflections has presentation

$$
\left\langle r_{1}, r_{2}, r_{3} \mid r_{1}^{2}=r_{2}^{2}=r_{3}^{2}=\left(r_{1} r_{2}\right)^{3}=\left(r_{2} r_{3}\right)^{3}=\left(r_{3} r_{1}\right)^{3}=1\right\rangle .
$$

If we think of $\mathbf{R}^{2}$ as the set of points $(u, v, w) \in \mathbf{R}^{3}$ with $u+v+w=0$, then this group acts on $\mathbf{R}^{2}$ by

$$
r_{1}(u, v, w)=(v, u, w), \quad r_{2}(u, v, w)=(u, w, v), \quad r_{3}(u, v, w)=(w+1, v, u-1) .
$$

In terms of these coordinates, the generalized cosine is given by

$$
h(u, v, w)=\left(e^{-2 \pi i u}+e^{-2 \pi i v}+e^{-2 \pi i w}, e^{-2 \pi i(u+v)}+e^{-2 \pi i(v+w)}+e^{-2 \pi i(u+w)}\right) .
$$

(Note that while the two components of $h$ are complex-valued, the relation $u+v+$ $w=0$ insures one is the complex conjugate of the other, so $h$ is really a function from $\mathbf{R}^{2}$ to $\mathbf{R}^{2}$.) Then the polynomials $P_{k}$ defined by (3) can be written as complexvalued functions of a complex variable $z$ (i.e., let $z$ be the first component of $h(\mathbf{x})$, and write the first component of $h(k \mathbf{x})$ in terms of $z$ ): they are polynomials in $z$ and $\bar{z}$. The first three are

$$
P_{1}(z, \bar{z})=z, \quad P_{2}(z, \bar{z})=z^{2}-2 \bar{z}, \quad P_{3}(z, \bar{z})=z^{3}-3 z \bar{z}+3 .
$$

The Chebyshev polynomials $T_{k}(x)$ are orthogonal with respect to the density $\left(1-x^{2}\right)^{-1 / 2}$ on the interval $[-1,1]$. The generalized Chebyshev polynomials satisfy a corresponding relation (Theorem 6.1 ). In proving this it is convenient to introduce a set of polynomial functions from $\mathbf{R}^{n}$ to $\mathbf{R}$, which we call orthogonal polynomials: the components of the folding polynomials $P_{k}$ are instances of them. The orthogonal polynomials are discussed in $\S 5$. The recurrence formula $T_{k}(x)=2 x T_{k-1}(x)-$ $T_{k-2}(x)$ can also be generalized (Theorem 6.2).

Besides the classical Chebyshev polynomials, there have been polynomials defined by several other authors $[\mathbf{5}, \mathbf{7}, \mathbf{8}, \mathbf{1 0}]$ that are special cases of the orthogonal polynomials or folding polynomials. These cases are noted in $\S 7$, where we give the function $h$ in explicit form for most foldable figures.

The paper by Dunkl [4] also studies orthogonal polynomials related to reflection groups, but there the object is to define "spherical harmonics" on the unit sphere in $\mathbf{R}^{n}$ and the reflection groups involved are finite. In our study, the polynomials arise from folding $\mathbf{R}^{n}$ into a compact fundamental domain and the groups must be infinite.

One aspect of the generalized Chebyshev polynomials not discussed here is their occurrence as solutions to differential equations. This topic, as well as the generalization of the Chebyshev polynomials of the second kind, will be treated in a forthcoming paper. 
2. Foldable figures and reflection groups. We first give a precise definition of a foldable figure.

DEFINITION. Let $F$ be a compact connected subset of $\mathbf{R}^{n}$ that is the closure of its interior. We call $F$ a foldable figure if there is a set $S$ of hyperplanes that cut $F$ into finitely many congruent subfigures $F_{1}, F_{2}, \ldots, F_{k}$, each similar to $F$, so that reflection in any of the hyperplanes in $S$ bounding $F_{i}$ takes it into some $F_{j}$.

THEOREM 2.1. A foldable figure in $\mathbf{R}^{n}$ is a convex polytope that tessellates $\mathbf{R}^{n}$ by reflections in hyperplanes.

PrOOF. Let $F$ be foldable: by hypothesis we can subdivide it by hyperplanes into congruent subfigures $F_{1}, \ldots, F_{k}$, each similar to $F$, so that reflection in any of the hyperplanes takes each subfigure onto another. Since $F_{1}$ is similar to $F$, it is also foldable and we can subdivide it into $k$ subfigures. Further, we can use reflection in the first set of hyperplanes to extend this subdivision into a division of $F$ into $k^{2}$ subfigures. Since every dihedral angle involved must be a submultiple of $\pi$, it follows that this subdivision of $F$ is also by hyperplanes. Continuing in this way, we have for any integer $p$ a subdivision by hyperplanes of $F$ into $k^{p}$ subfigures, and some sequence of reflections in these hyperplanes takes any of the subfigures into any other.

Now let $x$ be an interior point of $F$. Let $d$ be the distance from $x$ to the boundary of $F$. Continue the subdivision of $F$ until the subfigures have diameter less than $d$ : then the subfigure $F^{\prime}$ containing $x$ is entirely in the interior of $F$. But then $F^{\prime}$ is a convex polytope, since it is bounded by hyperplanes. Further, $F$ is tessellated by copies of $F^{\prime}$. Since $F$ is similar to $F^{\prime}$ we can tessellate a larger version of $F$ with copies of $F$, and by repeating this procedure tessellate all of $\mathbf{R}^{n}$.

We digress to develop some of the theory of reflection groups. Think of $\mathbf{R}^{n}$ as endowed with its usual inner product. Let $G$ be an $n$-dimensional reflection group, i.e. a discrete group of isometries of $\mathbf{R}^{n}$ generated by reflections through hyperplanes. The theory of such groups is well known: two convenient references are Bourbaki [1] and Coxeter [2]. In particular, such a group has a presentation of the form

$$
G=\left\langle r_{i}, 1 \leq i \leq k \mid\left(r_{i} r_{j}\right)^{m_{i j}}=1,1 \leq i \leq j \leq k\right\rangle
$$

for positive integers $m_{i j}$ such that $m_{i i}=1$ for all $i$ and $m_{i j} \geq 2$ for $i \neq j\left(m_{i j}=\infty\right.$ is allowed, in which case the corresponding relation is dropped out). Geometrically, the $r_{i}$ are reflections that generate $G$. This presentation (1) is called a Coxeter presentation (see Hiller [6]). Such a presentation can be represented by a graph $\Gamma$ that has one node for each generator and an edge from the node representing $r_{i}$ to that representing $r_{j}$ labeled with the integer $m_{i j}$. If $m_{i j}=2$ the edge is omitted (this is the case where $r_{i}$ and $r_{j}$ commute): the label $m_{i j}=3$, being the most common, is usually omitted. The labeled graph $\Gamma$ is the Coxeter graph of $G$.

The direct product of reflection groups is again a reflection group, and it is easy to see that the Coxeter graph of the product is the disjoint union of the Coxeter graphs of the factors. A reflection group is called irreducible if it is not a product of reflection groups: a reflection group is irreducible if and only if its Coxeter graph is connected.

If $G$ is an $n$-dimensional reflection group and $O_{n}$ is the group of linear isometries of $\mathbf{R}^{n}$ (i.e. those that fix the origin), then there is a homomorphism $U: G \rightarrow O_{n}$ 
given by

$$
U(g)(\mathbf{x})=g(\mathbf{x})-g(\mathbf{0}), \quad g \in G, \mathbf{x} \in \mathbf{R}^{n}
$$

(cf. [1, V, no. 3.6]). The group $G$ is called essential if the action of $U(G)$ on $\mathbf{R}^{n}$ fixes only the origin. Elements of the kernel of $U$ are called translations. The translations form a torsion-free abelian subgroup of $G$ : if $G$ is essential, this subgroup is either trivial or of rank $n$.

An $n$-dimensional reflection group $G$ has a fundamental region, i.e. a closed set $D$ such that no element of $G$ takes a point of $D$ to another, and such that for any $\mathbf{x} \in \mathbf{R}^{n}$ there is some $g \in G$ with $g(\mathbf{x}) \in D$. In fact, the Coxeter graph $\Gamma$ of $G$ can be interpreted in terms of the shape of $D$. The nodes of $\Gamma$ correspond to the faces of $D$. If two nodes are unconnected, then the corresponding faces intersect orthogonally. If two nodes are connected by an edge labeled $m$, then the corresponding faces intersect at an angle $\pi / m$. If $G$ is irreducible, then $D$ is a simplex or simplicial cone: otherwise, it is the product of fundamental regions of its irreducible factors (cf. $[2, \S 11.2])$.

If $F$ is a foldable figure, it is a fundamental region for the group generated by the reflections through its bounding hyperplanes $[\mathbf{1}, \mathrm{V}$, no. 3.3 , Théorème 2$]$. The fundamental regions of reflection groups are characterized in $[\mathbf{1}, \mathrm{V}$, no. 3.9]: for the fundamental region to be compact, the group must be essential and without fixed points. Thus we have the following result.

THEOREM 2.2. There is a one-to-one correspondence between foldable figures and reflection groups that are essential and without fixed points.

3. Root systems. Reflection groups can be classified by using the theory of root systems. This theory is developed in many references $($ e.g. $[\mathbf{1}, \mathbf{6}, \mathbf{1 2}])$ : we shall follow most closely the notation of $[\mathbf{1}]$. For any nonzero vector $\boldsymbol{\alpha}$ of $\mathbf{R}^{n}$, reflection in the hyperplane through the origin orthogonal to $\alpha$ is given by

$$
s_{\boldsymbol{\alpha}}(\mathbf{x})=\mathbf{x}-\frac{2 \mathbf{x} \cdot \boldsymbol{\alpha}}{\boldsymbol{\alpha} \cdot \boldsymbol{\alpha}} \boldsymbol{\alpha} .
$$

A root system can be defined as a finite subset $R$ of $\mathbf{R}^{n}$ satisfying the following properties:

(i) $R$ generates $\mathbf{R}^{n}$ as a vector space.

(ii) If $\boldsymbol{\alpha}, \boldsymbol{\beta} \in R$, then $s_{\boldsymbol{\alpha}}(\boldsymbol{\beta}) \in R$.

(iii) If $\alpha, k \alpha \in R$, then $k= \pm 1$.

(iv) If $\boldsymbol{\alpha}, \boldsymbol{\beta} \in R$, then $(2 \boldsymbol{\alpha} \cdot \boldsymbol{\beta}) /(\boldsymbol{\beta} \cdot \boldsymbol{\beta}) \in \mathbf{Z}$.

(This is a reduced root system in Bourbaki's terminology.) Property (iv) can be stated more succinctly as follows. For any root $\boldsymbol{\alpha}$, let

$$
\alpha^{\vee}=\frac{2 \alpha}{\alpha \cdot \alpha}
$$

( $\boldsymbol{\alpha}^{\vee}$ is called a coroot). Then (iv) requires that $\boldsymbol{\alpha} \cdot \boldsymbol{\beta}^{\vee} \in \mathbf{Z}$ for all $\boldsymbol{\alpha}, \boldsymbol{\beta} \in R$. The Weyl group of $R$ is the group $W$ generated by the reflections $s_{\boldsymbol{\alpha}}$ for $\boldsymbol{\alpha} \in R$ : it is always finite.

Any root system $R$ has a base, i.e. a linearly independent subset $B$ such that any root can be written as a linear combination of elements of $B$ with all positive or all negative coefficients. Corresponding to any base $B=\left\{\boldsymbol{\alpha}_{1}, \ldots, \boldsymbol{\alpha}_{n}\right\}$ is a Weyl 


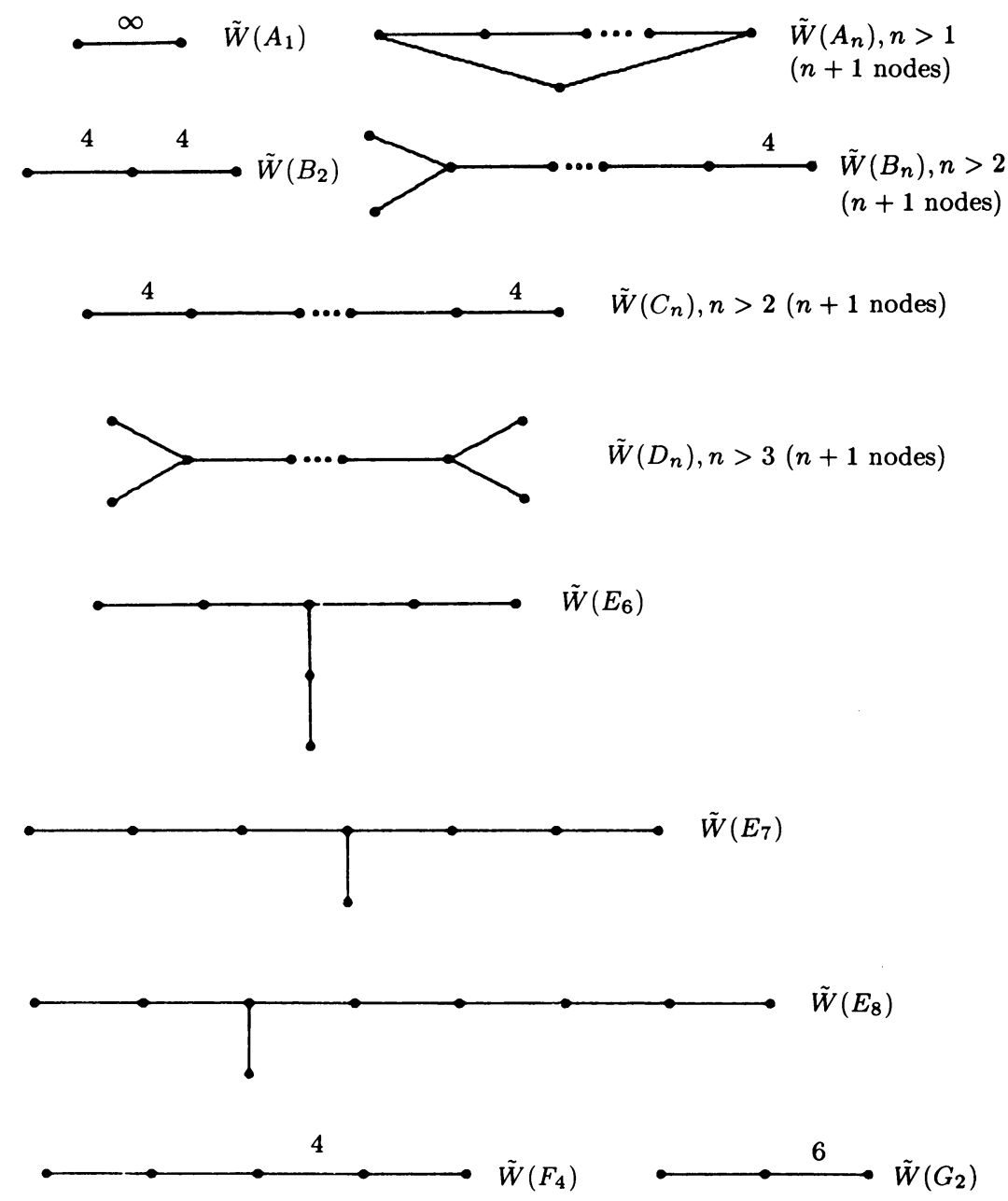

FIGURE 1

chamber $C=\left\{\mathbf{x} \in \mathbf{R}^{n} \mid \mathbf{x} \cdot \boldsymbol{\alpha}_{i}>0\right.$ for all $\left.i\right\}$. Then $\bar{C}$ is a fundamental region (necessarily noncompact) for the reflection group $W$.

For any root $\boldsymbol{\alpha}$ and integer $k$, there is a hyperplane

$$
L_{\boldsymbol{\alpha}, k}=\left\{\mathbf{x} \in \mathbf{R}^{n} \mid \boldsymbol{\alpha} \cdot \mathbf{x}=k\right\} .
$$

If we expand $W$ by adding the reflections in these hyperplanes, we obtain an infinite group $\tilde{W}$, called the affine Weyl group of $R$. Now the reflection through the hyperplane $L_{\boldsymbol{\alpha}, k}$ is given by

$$
s_{\boldsymbol{\alpha}, k}(\mathbf{x})=\mathbf{x}-\frac{2(\boldsymbol{\alpha} \cdot \mathbf{x}-k)}{\boldsymbol{\alpha} \cdot \boldsymbol{\alpha}} \boldsymbol{\alpha}=s_{\boldsymbol{\alpha}}(\mathbf{x})+k \boldsymbol{\alpha}^{\vee} .
$$

From this it follows that

$$
s_{\boldsymbol{\alpha}, k} \circ s_{\boldsymbol{\beta}, l}(\mathbf{x})=s_{\boldsymbol{\alpha}} \circ s_{\boldsymbol{\beta}}(\mathbf{x})+l s_{\boldsymbol{\alpha}}(\boldsymbol{\beta})^{\vee}+k \boldsymbol{\alpha}^{\vee}
$$

for roots $\boldsymbol{\alpha}, \boldsymbol{\beta}$ and integers $k, l$. We then have the following result. 
THEOREM 3.1. The affine Weyl group of a root system $R$ is a semidirect product $W \ltimes Q\left(R^{\vee}\right)$, where $W$ is the Weyl group of $R$ and $Q\left(R^{\vee}\right)$ is the abelian group generated by the coroots. Further, $Q\left(R^{\vee}\right)$ is the subgroup of translations of $\tilde{W}$ and $W$ is the isotropy group of the origin.

PROOF. The action of $W$ on $R$ extends to an action of $W$ on $Q\left(R^{\vee}\right)$. That $\tilde{W}$ is a semidirect product then follows from (1). If we think of $\tilde{W}$ as a reflection group, then the homomorphism $U$ of the last section evidently sends $s_{\boldsymbol{\alpha}, k}$ to $s_{\boldsymbol{\alpha}}$ : so $U$ has kernel $Q\left(R^{\vee}\right)$ and the second statement follows.

Given root systems $R_{1}$ in $\mathbf{R}^{n}$ and $R_{2}$ in $\mathbf{R}^{m}$, there is a root system $R_{1} \cup R_{2}$ in $\mathbf{R}^{n} \oplus \mathbf{R}^{m} \cong \mathbf{R}^{n+m}$, called the direct sum. The (affine) Weyl group of $R_{1} \cup R_{2}$ is the direct product of the (affine) Weyl groups of the factors. A root system is called irreducible if it is not a direct sum: from the well-known classification of irreducible root systems we have the following result [1, VI, no. 4.3, Théorème 4].

THEOREM 3.2. The only irreducible affine Weyl groups are $\tilde{W}\left(A_{n}\right), n \geq 1$, $\tilde{W}\left(B_{n}\right), n \geq 2, \tilde{W}\left(C_{n}\right), n \geq 3, \tilde{W}\left(D_{n}\right), n \geq 4, \tilde{W}\left(E_{6}\right), \tilde{W}\left(E_{7}\right), \tilde{W}\left(E_{8}\right), \tilde{W}\left(F_{4}\right)$, and $\tilde{W}\left(G_{2}\right)$, with Coxeter diagrams as shown in Figure 1.

The significance of the preceding discussion for our study of foldable figures is made clear by the next result, which follows from [1, VI, no. 2.5, Proposition 8].

THEOREM 3.3. The reflection group corresponding to a foldable figure $F$ is the affine Weyl group of some root system.

In view of the remarks preceding Theorem 2.2 , we can regard a foldable figure as a fundamental region of some affine Weyl group $\tilde{W}$. Given a base for the corresponding root system, the Weyl chamber includes exactly one fundamental region of $\tilde{W}$ that contains the origin.

4. The generalized cosine. Starting with an affine Weyl group $\tilde{W}$, we seek a continuous coordinate change $h: \mathbf{R}^{n} \rightarrow \mathbf{R}^{n}$ such that

(i) $h(g(\mathbf{x}))=h(\mathbf{x})$ for $g \in \tilde{W}, \mathbf{x} \in \mathbf{R}^{n}$; and

(ii) $h(k \mathbf{x})$ is a polynomial in $h(\mathbf{x})$ for $k=1,2, \ldots$

By Theorem 3.1, $\tilde{W}$ can be written as a semidirect product $W \ltimes Q\left(R^{\vee}\right)$, where $R$ is a root system and $W$ the corresponding Weyl group (thought of as the isotropy group of the origin). Let $\boldsymbol{\alpha}_{1}, \ldots, \boldsymbol{\alpha}_{n}$ be a base for the root system $R$ : corresponding to this is a unique fundamental region containing the origin, which we denote $\Phi$. The weights associated with $R$ are defined to be the elements of the group

$$
P(R)=\left\{\mathbf{r} \in \mathbf{R}^{n} \mid \mathbf{r} \cdot \mathbf{s} \in \mathbf{Z} \text { for all } \mathbf{s} \in Q\left(R^{\vee}\right)\right\} .
$$

The lattice $P(R)$ has generators $\omega_{1}, \omega_{2}, \ldots, \omega_{n}$ defined by

$$
\omega_{j} \cdot \boldsymbol{\alpha}_{k}^{\vee}=\delta_{j k} .
$$

(The vectors $\omega_{k}$ are called fundamental weights.) Let $\omega_{0}=-\omega_{1}-\cdots-\omega_{n}$ : then any element of $P(R)$ can be written as a sum (with positive integer coefficients) of $\omega_{0}, \ldots, \omega_{n}$.

We denote the position vector in $\mathbf{R}^{n}$ by $\mathbf{x}$ and for $k=0, \ldots, n$ set

$$
s_{k}(\mathbf{x})=e^{-2 \pi i \mathbf{x} \cdot \omega_{k}} \text {. }
$$

Then for any $\mathbf{r} \in P(R), e^{-2 \pi i \mathbf{x} \cdot \mathbf{r}}$ can be written as a product of $s_{0}, \ldots, s_{n}$. Also $s_{0} s_{1} \cdots s_{n}=1$. The proof of the next lemma is then straightforward. 
LEMMA 4.1. Let $f: P(R) \rightarrow \mathbf{R}$ have finite support and be invariant under $W$. Then the Fourier transform of $f$,

$$
\mathscr{F}(f)(\mathbf{x})=\sum_{\mathbf{r} \in P(R)} f(\mathbf{r}) e^{-2 \pi i \mathbf{x} \cdot \mathbf{r}},
$$

is invariant as a function of $\mathbf{x}$ under $\tilde{W}$. Moreover, $\mathscr{F}(f)$ is a polynomial in $s_{0}, \ldots, s_{n}$.

Note that the Fourier transform used here involves a sum rather than an integral. While we could interpret this as the Fourier transform of a distribution (as in Schwartz [13]), we shall instead derive those of its properties we need.

The set $\Psi$ of functions $f: P(R) \rightarrow \mathbf{R}^{n}$ with finite support and invariant under $W$ forms a vector space. A natural basis for this space is the set of characteristic functions

$$
\chi_{\phi}(\mathbf{x})= \begin{cases}1 & \text { if } \mathbf{x} \in \phi, \\ 0 & \text { if } \mathbf{x} \notin \phi,\end{cases}
$$

where $\phi$ is the orbit of some point in $P(R)$ under $W$. Let $\Omega$ denote the set of such orbits. For $\phi \in \Omega$ we denote the number of points in $\phi$ by $|\phi|$. From [1, VI, no. 3.4, Théorème 1] we have the following result.

THEOREM 4.2. For $k=1, \ldots, n$ let $\phi_{k}$ be the orbit generated by the fundamental weight $\omega_{k}$ of the root system of $W$, and let $y_{k}$ be the Fourier transform of $\chi_{\phi_{k}}$. Then for any $f \in \Psi, \mathscr{F}(f)$ can be written as a polynomial in $\mathbf{y}=\left(y_{1}, \ldots, y_{n}\right)$.

Thus the Fourier transform gives a one-to-one correspondence between $\Psi$ and the set of polynomials in $y_{1}, \ldots, y_{n}$. The theorem says that any polynomial in $s_{0}, \ldots, s_{n}$ invariant under the action of $W$ on $P(R)$ can be written as a polynomial in $y_{1}, \ldots, y_{n}$.

DEFINITION. For an affine Weyl group $\tilde{W}$, let $y_{1}, \ldots, y_{n}$ be as in Theorem 4.2. We define the generalized cosine $h: \mathbf{R}^{n} \rightarrow \mathbf{R}^{n}$ associated with $\tilde{W}$ by

$$
h(\mathbf{x})=\left(y_{1}(\mathbf{x}), y_{2}(\mathbf{x}), \ldots, y_{n}(\mathbf{x})\right) .
$$

THEOREM 4.3. The generalized cosine $h$ is invariant under $\tilde{W}$, and $h(k \mathbf{x})$ is a polynomial in $h(\mathbf{x})$ for integer $k \geq 1$. Further, $h$ is a homeomorphism of $\Phi$ onto its image, and can be assumed orientation-preserving.

ProOF. That $h$ is invariant under $\tilde{W}$ follows from Lemma 4.1. Also

$$
y_{j}(k \mathbf{x})=\sum_{\mathbf{r} \in P(R)} \chi_{\phi_{j}}(\mathbf{r}) e^{-2 \pi i k \mathbf{x} \cdot \mathbf{r}}=\sum_{\mathbf{r} \in P(R)} f(\mathbf{r}) e^{-2 \pi i \mathbf{x} \cdot \mathbf{r}},
$$

where $f(\mathbf{r})=\chi_{\phi_{j}}(\mathbf{r} / k)$ if $\mathbf{r} / k \in P(R), f(\mathbf{r})=0$ otherwise. Thus $f \in \Psi$ and by Theorem $4.2 y_{j}(k \mathbf{x})$ is a polynomial in $h(\mathbf{x})$.

To see that $h$ is one-to-one from $\Phi$ onto its image, choose $\mathbf{x}^{\prime}, \mathbf{x}^{\prime \prime} \in \Phi$ such that $h\left(\mathbf{x}^{\prime}\right)=h\left(\mathbf{x}^{\prime \prime}\right)$. For $j=1, \ldots, n$, let

$$
a_{j}=e^{-2 \pi i \mathbf{x}^{\prime \prime} \cdot \omega_{j}}
$$

Now define

$$
p(\mathbf{x})=\prod_{g \in W} \sum_{j=1}^{n}\left(e^{-2 \pi i g(\mathbf{x}) \cdot \omega_{j}}-a_{j}\right)\left(e^{2 \pi i g(\mathbf{x}) \cdot \omega_{j}}-\bar{a}_{j}\right) .
$$


Then $p(\mathbf{x})$ is a polynomial in $s_{0}, \ldots, s_{n}$ and is invariant under the action of $W$ on $\mathbf{x}$. By Theorem $4.2, p(\mathbf{x})$ can be written as a polynomial in $\left(y_{1}, \ldots, y_{n}\right)=h(\mathbf{x})$. Since $h\left(\mathbf{x}^{\prime}\right)=h\left(\mathbf{x}^{\prime \prime}\right)$, we have $p\left(\mathbf{x}^{\prime}\right)=p\left(\mathbf{x}^{\prime \prime}\right)=0$. Thus

$$
\sum_{j=1}^{n}\left(e^{-2 \pi i g\left(\mathbf{x}^{\prime}\right) \cdot \omega_{j}}-a_{j}\right)\left(e^{2 \pi i g\left(\mathbf{x}^{\prime}\right) \cdot \omega_{j}}-\bar{a}_{j}\right)=0
$$

for some $g \in W$. Since each term in this sum is nonnegative, we have for $j=1, \ldots, n$

$$
e^{-2 \pi i g\left(\mathbf{x}^{\prime}\right) \cdot \omega_{j}}-a_{j}=e^{-2 \pi i g\left(\mathbf{x}^{\prime}\right) \cdot \boldsymbol{\omega}_{j}}-e^{-2 \pi i \mathbf{x}^{\prime \prime} \cdot \boldsymbol{\omega}_{j}}=0
$$

and thus

$$
e^{-2 \pi i\left[g\left(\mathbf{x}^{\prime}\right)-\mathbf{x}^{\prime \prime}\right] \cdot \omega_{j}}=1 .
$$

Since $\omega_{1}, \ldots, \omega_{n}$ is a basis for $P(R)$, we must have $g\left(\mathbf{x}^{\prime}\right)-\mathbf{x}^{\prime \prime} \in Q\left(R^{\vee}\right)$. Thus there exists an element $w$ of $\tilde{W}$ with $w\left(\mathbf{x}^{\prime}\right)=\mathbf{x}^{\prime \prime}$. Since $\mathbf{x}^{\prime}, \mathbf{x}^{\prime \prime} \in \Phi$, we have $x^{\prime}=x^{\prime \prime}$.

Clearly $h$ is continuous: since $\Phi$ is compact, $h$ is a homeomorphism of $\Phi$ onto $h(\Phi)$. Then $h$ is orientation-preserving or orientation-reversing on $\Phi$. In the latter case, choose an orientation-reversing element $g \in W$ (i.e. the product of an odd number of reflections) and replace $h$ by $h^{\prime}=h \circ g$ and $\Phi$ by $\Phi^{\prime}=g^{-1}(\Phi)$ : then $h^{\prime}$ has all the properties of $h$ and preserves orientation.

Let $\Delta=h(\Phi)$. We call $\Delta$ the natural domain for $\tilde{W}$. Since $h$ is continuous, $\Delta$ is compact.

5. The orthogonal polynomials. Consider the Jacobian determinant

$$
D=\frac{\partial\left(y_{1}, \ldots, y_{n}\right)}{\partial\left(u_{1}, \ldots, u_{n}\right)}
$$

of $h$, where we use the quantities $u_{k}=\mathbf{x} \cdot \boldsymbol{\omega}_{k}$ as coordinates. By the exponential nature of the functions $y_{k}$ of Theorem 4.2, the partial derivatives of $y_{1}, \ldots, y_{n}$ with respect to $u_{1}, \ldots, u_{n}$ are polynomials in $s_{0}, \ldots, s_{n}$. The quantity $D$ is not invariant under $\tilde{W}$ since it changes sign when going from one fundamental region to an adjacent one, but $D^{2}$ is invariant under $\tilde{W}$. It follows that $D^{2}$ can be written as a polynomial in $y_{1}, \ldots, y_{n}$; we denote this polynomial by $v$.

DEFINITION. For each $\phi \in \Omega$, define the orthogonal polynomial $j_{\phi}$ associated with $\tilde{W}$ by

$$
j_{\phi}(h(\mathbf{x}))=\mathscr{F}\left(\chi_{\phi}\right)(\mathbf{x}), \quad \mathbf{x} \in \mathbf{R}^{n} .
$$

This terminology is justified by the next theorem.

THEOREM 5.1. Let $\delta(\mathbf{y})=(v(\mathbf{y}))^{-1 / 2}$. Let $V$ be the volume of $\Phi$ relative to the coordinates $u_{1}, \ldots, u_{n}$. Then for $\psi, \phi \in \Omega$,

$$
\int_{\Delta} j_{\phi}(\mathbf{y}) \overline{j_{\psi}(\mathbf{y})} \delta(\mathbf{y}) d y_{1} \cdots d y_{n}= \begin{cases}0 & \text { if } \phi \neq \psi, \\ V|\phi| & \text { if } \phi=\psi\end{cases}
$$

PROOF. First make the change of coordinates $\mathbf{y}=h(\mathbf{x})$; the integral becomes

$$
\begin{aligned}
\int_{\Delta} j_{\phi}(h(\mathbf{x})) \overline{j_{\psi}(h(\mathbf{x}))} \delta(\mathbf{y}) d y_{1} \cdots d y_{n} & =\int_{\Phi} \sum_{\mathbf{r} \in \phi} \sum_{\mathbf{s} \in \psi} e^{-2 \pi i \mathbf{x} \cdot \mathbf{r}} e^{2 \pi i \mathbf{x} \cdot \mathbf{8}} d u_{1} \cdots d u_{n} \\
& =\int_{\Phi} F(\mathbf{x}) d u_{1} \cdots d u_{n}
\end{aligned}
$$


where

$$
F(\mathbf{x})=\sum_{\mathbf{r} \in \phi} \sum_{\mathbf{s} \in \psi} e^{2 \pi i \mathbf{x} \cdot(\mathbf{s}-\mathbf{r})} .
$$

Let $I$ denote the value of this integral.

For integer $N$, define

$$
S(N)=\left\{\mathbf{x} \mid-N \leq u_{k} \leq N, k=1, \ldots, n\right\} .
$$

The volume of $S(N)$ relative to the coordinates $u_{1}, \ldots, u_{n}$ is $(2 N)^{n}$. The integrand in (1) is a sum of exponential terms, the exponent in each term being a linear combination of $u_{1}, \ldots, u_{n}$ with integer coefficients. If $\phi \neq \psi$, then no term has all zero coefficients in the exponent; if $\phi=\psi$, then all the coefficients are zero in just those terms where $\mathbf{r}=\mathbf{s}$. There are exactly $|\phi|$ of these terms. Thus

$$
K(N)=\int_{S(N)} F(\mathbf{x}) d u_{1} \cdots d u_{n}= \begin{cases}0 & \text { if } \phi \neq \psi \\ (2 N)^{n}|\phi| & \text { if } \phi=\psi\end{cases}
$$

Let $\underline{M}(N)$ be the number of fundamental regions entirely contained in $S(N)$, $\bar{M}(N)$ the number of fundamental regions that intersect $S(N)$. We estimate $\underline{M}$ and $\bar{M}$ as follows. Let $\rho$ be the metric on $\mathbf{R}^{n}$ given by

$$
\rho\left(\mathbf{x}, \mathbf{x}^{\prime}\right)=\sqrt{\left(u_{1}-u_{1}^{\prime}\right)^{2}+\cdots+\left(u_{n}-u_{n}^{\prime}\right)^{2}} .
$$

(The elements of $\tilde{W}$ may not be isometries with respect to $\rho$.) Set

$$
d=\sup \left\{\operatorname{diam}_{\rho} w(\Phi) \mid w \in W\right\}
$$

Then

$$
2^{n}(N-d)^{n} / V \leq \underline{M}(N) \leq \bar{M}(N) \leq 2^{n}(N+d)^{n} / V .
$$

The integrand $F(\mathbf{x})$ is invariant under $\tilde{W}$, so the integral takes the same value no matter which fundamental region is integrated over. Note also $|F(\mathbf{x})| \leq|\phi||\psi|$, so for any subset $E$ of a fundamental region we have

$$
\left|\int_{E} F(\mathbf{x}) d u_{1} \cdots d u_{n}\right| \leq|\phi||\psi| V
$$

Therefore

$$
|K(N)-\underline{M}(N) I| \leq(\bar{M}-\underline{M})|\phi||\psi| V,
$$

and hence, using (2),

$$
\left|(2 N)^{-n} K(N)-(2 N)^{-n} \underline{M}(N) I\right| \leq\left(\left(\frac{N+d}{N}\right)^{n}-\left(\frac{N-d}{N}\right)^{n}\right)|\phi||\psi| .
$$

Taking the limit as $N \rightarrow \infty$, we have $I=0$ if $\phi \neq \psi$ and $I=V|\phi|$ if $\phi=\psi$.

THEOREM 5.2. Let $p, q$ be polynomials and $f, g \in \Psi$ such that $p \circ h=\mathscr{F}(f)$, $q \circ h=\mathscr{F}(g)$. Then

$$
\int_{\Delta} p(\mathbf{y}) \overline{q(\mathbf{y})} \delta(\mathbf{y}) d y_{1} \cdots d y_{n}=V \sum_{\mathbf{r} \in P(R)} f(\mathbf{r}) \overline{g(\mathbf{r})}
$$


PROOF. Expand $f$ and $g$ in series of characteristic functions:

$$
f=\sum_{\phi \in \Omega} a_{\phi} \chi_{\phi}, \quad g=\sum_{\phi \in \Omega} b_{\phi} \chi_{\phi} .
$$

Then

Thus

$$
p=\sum_{\phi \in \Omega} a_{\phi} j_{\phi}, \quad q=\sum_{\phi \in \Omega} b_{\phi} j_{\phi}
$$

$$
\begin{aligned}
\int_{\Delta} p(\mathbf{y}) \overline{q(\mathbf{y})} \delta(\mathbf{y}) d y_{1} \cdots d y_{n} & =\sum_{\phi \in \Omega} a_{\phi} \bar{b}_{\phi} \int_{\Delta} j_{\phi}(\mathbf{y}) \overline{j_{\phi}(\mathbf{y})} \delta(\mathbf{y}) d y_{1} \cdots d y_{n} \\
& =\sum_{\phi \in \Omega} a_{\phi} \bar{b}_{\phi}|\phi| v \\
& =V \sum_{\phi \in \Omega} \sum_{\mathbf{r} \in \phi} f(\mathbf{r}) \overline{g(\mathbf{r})}=V \sum_{\mathbf{r} \in P(R)} f(\mathbf{r}) \overline{g(\mathbf{r})} .
\end{aligned}
$$

THEOREM 5.3. The set of polynomials $\left\{j_{\phi} \mid \phi \in \Omega\right\}$ is complete in the space $C(\Delta)$ of continuous functions on $\Delta$.

PROOF. If not, by the Weierstrass Approximation Theorem [3, p. 274] there exists a nonzero polynomial $q$ which cannot be approximated by the polynomials $j_{\phi}$. Since $\Delta$ is compact, $C(\Delta)$ contains all polynomials and is contained in $L^{2}(\Delta)$. So $q$ must contain a component polynomial $p$ orthogonal to the $j_{\phi}$ :

$$
\int_{\Delta} p(\mathbf{y}) \overline{j_{\phi}(\mathbf{y})} \delta(\mathbf{y}) d y_{1} \cdots d y_{n}=0
$$

for all $\phi \in \Omega$. Let $f \in \Psi$ be such that $p \circ h=\mathscr{F}(f)$. Then by Theorem 5.2 we have

$$
\sum_{\mathbf{r} \in P(R)} f(\mathbf{r}) \overline{\chi_{\phi}(\mathbf{r})}=0
$$

for all $\phi \in \Omega$. But this implies $f=0$ and hence $p=0$.

6. Generalized Chebyshev polynomials. We retain the notation of the last two sections.

DEFINITION. For integer $k \geq 1$, the generalized Chebyshev polynomials (or folding polynomials) associated with $\Phi$ are the polynomial functions $P_{k}: \mathbf{R}^{n} \rightarrow \mathbf{R}^{n}$ defined by

$$
P_{k}(h(\mathbf{x}))=h(k \mathbf{x}), \quad \mathbf{x} \in \mathbf{R}^{n} .
$$

Of course the components of the generalized Chebyshev polynomials are orthogonal polynomials in the sense of the previous section. In fact, for any orbit $\phi$ in $\Omega$, $k \phi=\{k \mathbf{x} \in P(R) \mid \mathbf{x} \in \phi\}$ is also an orbit, and

$$
P_{k}=\left(j_{k \phi_{1}}, \ldots, j_{k \phi_{n}}\right),
$$

where $\phi_{1}, \ldots, \phi_{n}$ are as in the statement of Theorem 4.2. From the definitions it follows easily that

$$
j_{\phi} \circ P_{k}=j_{k \phi}
$$

for any positive integer $k$ and orbit $\phi \in \Omega$, and also that

$$
P_{k} \circ P_{l}=P_{k l}
$$

for any positive integers $k$ and $l$. 
On the other hand, it is not true that "most" orthogonal polynomials appear as components of the $P_{k}$, since the orbits of the form $k \phi_{j}$ are rather special. In fact, we can characterize such orbits as follows. Let $C$ be as in $\S 3$, so $\bar{C}$ is a fundamental region for $W$. Now any orbit $\phi \in \Omega$ has exactly one point $\mathbf{r} \in \bar{C}$. If we write

$$
\mathbf{r}=r_{1} \omega_{1}+\cdots+r_{n} \omega_{n}
$$

then it follows from the definitions of $C$ and the $\omega_{j}$ that $r_{i}$ is a nonnegative integer for all $i$. For $\phi$ to be of the form $k \phi_{j}, \mathbf{r}$ must have the form $k \omega_{j}$, i.e. $r_{i}=k \delta_{i j}$.

An orthogonality result for the generalized Chebyshev polynomials follows immediately from Theorem 5.1 . $k, l$,

THEOREM 6.1. Let $\delta(\mathbf{y}), \Delta$, and $V$ be as in Theorem 5.1. Then for integers

$$
\int_{\Delta} P_{k}(\mathbf{y}) \cdot \overline{P_{l}(\mathbf{y})} \delta(\mathbf{y}) d y_{1} \cdots d y_{n}=N \delta_{k l},
$$

where $N=V\left(\left|\phi_{1}\right|+\cdots+\left|\phi_{n}\right|\right)$.

As we see in the next result, each component of a generalized Chebyshev polynomial satisfies a recurrence formula.

THEOREM 6.2. Let $\phi_{k}=\left\{\mathbf{z}_{1}, \ldots, \mathbf{z}_{r}\right\}$ (so $r=\left|\phi_{k}\right|$ ), and let $\sigma_{1}, \ldots, \sigma_{r}$ be the elementary symmetric functions in the variables $e^{-2 \pi i \mathbf{z}_{1} \cdot \mathbf{x}}, \ldots, e^{-2 \pi i \mathbf{z}_{r} \cdot \mathbf{x}}$. Then for $m>r$,

$$
j_{m \phi_{k}}=\sigma_{1} j_{(m-1) \phi_{k}}-\sigma_{2} j_{(m-2) \phi_{k}}+\cdots+(-1)^{r+1} \sigma_{r} j_{(m-r) \phi_{k}} .
$$

PROOF. First note that

$$
\prod_{s=1}^{r}\left(t-e^{-2 \pi i \mathbf{z}_{s} \cdot \mathbf{x}}\right)=t^{r}-\sigma_{1} t^{r-1}+\cdots+(-1)^{r} \sigma_{r} .
$$

Now substitute $t=e^{-2 \pi i z_{s} \cdot \mathbf{x}} ;$ since the left-hand side is zero, we have

$$
e^{-2 \pi i r \mathbf{z}_{s} \cdot \mathbf{x}}=\sigma_{1} e^{-2 \pi i(r-1) \mathbf{z}_{s} \cdot \mathbf{x}}-\sigma_{2} e^{-2 \pi i(r-2) \mathbf{z}_{s} \cdot \mathbf{x}}+\cdots+(-1)^{r+1} \sigma_{r} .
$$

Next multiply both sides by $e^{-2 \pi i(m-r) \mathbf{z}_{s} \cdot \mathbf{x}}$ and sum over $s$ to obtain the conclusion.

REMARK. The functions $\sigma_{1}, \ldots, \sigma_{r}$ of the theorem can be expressed in terms of $j_{\phi_{k}}, j_{2 \phi_{k}}, \ldots, j_{r \phi_{k}}$. First note that $j_{m \phi_{k}}$ is the $m$ th power-sum symmetric function in the variables $e^{-2 \pi i \mathbf{z}_{1} \cdot \mathbf{x}}, \ldots, e^{-2 \pi i \mathbf{z}_{r} \cdot \mathbf{x}}$. Since this is a symmetric function, it can be written as a polynomial in the elementary symmetric functions $\sigma_{1}, \ldots, \sigma_{r}$. Conversely, the elementary symmetric functions can be written as polynomials in the power-sum symmetric functions. Using the formula of Macdonald [9, p. 20], we have

$$
\sigma_{s}=\frac{1}{s !} \operatorname{det}\left[\begin{array}{cccc}
j_{\phi_{k}} & 1 & \cdots & 0 \\
j_{2 \phi_{k}} & j_{\phi_{k}} & \cdots & 0 \\
\vdots & \vdots & \ddots & \vdots \\
j_{(s-1) \phi_{k}} & j_{(s-2) \phi_{k}} & \cdots & s-1 \\
j_{s \phi_{k}} & j_{(s-1) \phi_{k}} & \cdots & j_{\phi_{k}}
\end{array}\right]
$$


7. Examples. In this section we exhibit some explicit formulas for the generalized cosines for foldable figures as given by the construction of $\S 4$. We confine ourselves to cases where the affine Weyl group is irreducible, i.e. where the foldable figure is a simplex, since the generalized cosine (and hence the orthogonal and folding polynomials) for a product can be obtained immediately from those for the factors. We give such formulas for all the irreducible affine Weyl groups as classified in Theorem 3.2 , save for the three exceptional ones $\tilde{W}\left(E_{6}\right), \tilde{W}\left(E_{7}\right)$ and $\tilde{W}\left(E_{8}\right)$, where they seem too complicated to be of interest. We also point out some additional families of polynomials associated with certain affine Weyl groups.

In the following, rather than the coordinates $u_{1}, \ldots, u_{n}$ of $\S 5$ we use coordinates $v_{1}, \ldots, v_{n}$ which give a more symmetrical expression for $h$. In each case we shall give the relation between $u_{i}, \ldots, u_{n}$ and $v_{1}, \ldots, v_{n}$. As above, $\sigma_{j}$ denotes the $j$ th elementary symmetric function.

For the group $\tilde{W}\left(A_{n}\right)$, define coordinates $v_{1}, \ldots, v_{n}$ as follows. Let $\mathbf{e}_{0}, \mathbf{e}_{1}, \ldots, \mathbf{e}_{n}$ be orthogonal unit vectors in $\mathbf{R}^{n+1}$. Think of $\mathbf{R}^{n}$ as the hyperplane in $\mathbf{R}^{n+1}$ perpendicular to the vector $\mathbf{e}_{0}+\cdots+\mathbf{e}_{n}$. For $\mathbf{x} \in \mathbf{R}^{n}$, set $v_{j}=\mathbf{x} \cdot \mathbf{e}_{j}$, so that

$$
v_{0}=-\sum_{j=1}^{n} v_{j} \text {. }
$$

The generalized cosine $h$ for $\tilde{W}\left(A_{n}\right)$ is then given by

$$
h\left[\begin{array}{c}
v_{1} \\
\vdots \\
v_{n}
\end{array}\right]=\left[\begin{array}{c}
\sigma_{1}\left(e^{-2 \pi i v_{0}}, \ldots, e^{-2 \pi i v_{n}}\right) \\
\vdots \\
\sigma_{n}\left(e^{-2 \pi i v_{0}}, \ldots, e^{-2 \pi i v_{n}}\right)
\end{array}\right]
$$

Note that while the coordinates $y_{1}, \ldots, y_{n}$ of $h\left(v_{1}, \ldots, v_{n}\right)$ are complex-valued, $y_{j}=\bar{y}_{n+1-j}$. The coordinates $u_{1}, \ldots, u_{n}$ are given by $u_{1}=v_{1}, u_{j}=u_{j-1}+v_{j}$ for $j=2, \ldots, n$.

In particular, if $n=1$ we have $h\left(v_{1}\right)=2 \cos 2 \pi v_{1}$ and the generalized Chebyshev polynomials $P_{k}$ are related to the classical ones $T_{k}$ by $P_{k}(x)=2 T_{k}(x / 2)$. The generalized Chebyshev polynomials for the case $n=2$ appear in the work of Mittag and Stephen [10] on the Potts model, and the orthogonal polynomials for this case have been described by Koornwinder [7, 8]. The orthogonal polynomials associated with $\tilde{W}\left(A_{n}\right)$ have been described by Eier and Lidl [5].

For the group $\tilde{W}\left(C_{n}\right)$, let $\mathbf{e}_{1}, \ldots, \mathbf{e}_{n}$ be orthogonal unit vectors in $\mathbf{R}^{n}$ and set $v_{j}=\mathbf{x} \cdot \mathbf{e}_{j}$. Then

$$
h\left[\begin{array}{c}
v_{1} \\
\vdots \\
v_{n}
\end{array}\right]=\left[\begin{array}{c}
2 \sigma_{1}\left(\cos 2 \pi v_{1}, \ldots, \cos 2 \pi v_{n}\right) \\
\vdots \\
2^{n} \sigma_{n}\left(\cos 2 \pi v_{1}, \ldots, \cos 2 \pi v_{n}\right)
\end{array}\right] .
$$

The coordinates $u_{1}, \ldots, u_{n}$ are given by $u_{1}=v_{1}, u_{j}=u_{j-1}+v_{j}$ for $j=2, \ldots, n$.

For the group $\tilde{W}\left(B_{n}\right)$, the coordinates $v_{1}, \ldots, v_{n}$ are defined as for $\tilde{W}\left(C_{n}\right)$. We then have

$$
h\left[\begin{array}{c}
v_{1} \\
\vdots \\
v_{n}
\end{array}\right]=\left[\begin{array}{c}
2^{n} \cos \pi v_{1} \ldots \cos \pi v_{n} \\
2 \sigma_{1}\left(\cos 2 \pi v_{1}, \ldots, \cos 2 \pi v_{n}\right) \\
\vdots \\
2^{n-1} \sigma_{n-1}\left(\cos 2 \pi v_{1}, \ldots, \cos 2 \pi v_{n}\right)
\end{array}\right] .
$$


The coordinates $u_{1}, \ldots, u_{n}$ are given by $u_{1}=v_{1}, u_{j}=u_{j-1}+v_{j}$ for $j=2, \ldots, n-1$, and $u_{n}=\left(v_{1}+\cdots+v_{n}\right) / 2$. The orthogonal polynomials associated with $\tilde{W}\left(B_{2}\right)$ have been described in [ $\mathbf{7}$ and $\mathbf{8}$.

The fundamental region for $\tilde{W}\left(B_{2}\right)$ is a right isosceles triangle (recall the remarks preceding Theorem 2.2). Besides being foldable into $n^{2}$ copies of itself, this triangle can be folded into $2 n^{2}$ copies of itself. Thus for $\tilde{W}\left(B_{2}\right)$ besides the usual sequence $P_{1}, P_{2}, \ldots$ of folding polynomials there is an additional sequence $P_{\sqrt{2}}, P_{2 \sqrt{2}}, \ldots$ given by

$$
P_{k \sqrt{2}}\left(h\left(v_{1}, v_{2}\right)\right)=h\left(k v_{1}+k v_{2}, k v_{2}-k v_{1}\right) .
$$

The composition property $P_{\alpha} \circ P_{\beta}=P_{\alpha \beta}$ also applies to this extra sequence of polynomials.

For the group $\tilde{W}\left(D_{n}\right)$, the coordinates $v_{1}, \ldots, v_{n}$ are defined as for $\tilde{W}\left(C_{n}\right)$. Then

$$
h\left[\begin{array}{c}
v_{1} \\
\vdots \\
v_{n}
\end{array}\right]=\left[\begin{array}{c}
2^{n-1}\left(\cos \pi v_{1} \cdots \cos \pi v_{n}+\sin \pi v_{1} \cdots \sin \pi v_{n}\right) \\
2^{n-1}\left(\cos \pi v_{1} \cdots \cos \pi v_{n}-\sin \pi v_{1} \cdots \sin \pi v_{n}\right) \\
2 \sigma_{1}\left(\cos 2 \pi v_{1}, \ldots, \cos 2 \pi v_{n}\right) \\
\vdots \\
2^{n-2} \sigma_{n-2}\left(\cos 2 \pi v_{1}, \ldots, \cos 2 \pi v_{n}\right)
\end{array}\right] .
$$

The coordinates $u_{1}, \ldots, u_{n}$ are given by $u_{1}=v_{1}, u_{j}=u_{j-1}+v_{j}$ for $j=2, \ldots, n-2$, $u_{n-1}=\left(v_{1}+\cdots+v_{n}\right) / 2$, and $u_{n}=\left(v_{1}+\cdots+v_{n-1}-v_{n}\right) / 2$.

The group $\tilde{W}\left(G_{2}\right)$ has as fundamental region a triangle with angles of 30,60 , and 90 degrees. The coordinates $v_{0}, v_{1}$ and $v_{2}$ are defined as for $\tilde{W}\left(A_{2}\right)$. We then have

$$
h\left[\begin{array}{l}
v_{1} \\
v_{2}
\end{array}\right]=\left[\begin{array}{c}
2\left(\cos 2 \pi v_{0}+\cos 2 \pi v_{1}+\cos 2 \pi v_{2}\right) \\
2\left(\cos 2 \pi\left(v_{0}-v_{1}\right)+\cos 2 \pi\left(v_{1}-v_{2}\right)+\cos 2 \pi\left(v_{2}-v_{0}\right)\right)
\end{array}\right] .
$$

The 30-60-90 triangle is similar to the right isosceles triangle in that, besides being foldable into $n^{2}$ copies of itself, it can be folded into $3 n^{2}$ copies of itself. Thus, associated with $\tilde{W}\left(G_{2}\right)$ there is an extra sequence of folding polynomials $P_{\sqrt{3}}, P_{2 \sqrt{3}}, \ldots$ given by

$$
P_{k \sqrt{3}}\left(h\left(v_{1}, v_{2}\right)\right)=h\left(k v_{1}-k v_{2}, k v_{2}-k v_{0}\right) .
$$

This extra sequence of polynomials satisfies the usual composition property.

For the group $\tilde{W}\left(F_{4}\right)$ we define $v_{1}, \ldots, v_{4}$ as for $\tilde{W}\left(C_{4}\right)$. Then

$$
h\left[\begin{array}{c}
v_{1} \\
v_{2} \\
v_{3} \\
v_{4}
\end{array}\right]=\left[\begin{array}{c}
16 \cos \pi v_{1} \cdots \cos \pi v_{4}+2 \sigma_{1} \\
4 \sigma_{2} \\
8 \sigma_{3}+\left(2 \sigma_{1}-1\right) \cos \pi v_{1} \cdots \cos \pi v_{4} \\
16 \sigma_{1} \sigma_{3}-16 \sigma_{2}-64 \sigma_{4}
\end{array}\right]
$$

where $\sigma_{j}=\sigma_{j}\left(\cos 2 \pi v_{1}, \ldots, \cos 2 \pi v_{4}\right)$.

The group $\tilde{W}\left(F_{4}\right)$, like $\tilde{W}\left(B_{2}\right)$ and $\tilde{W}\left(G_{2}\right)$, gives rise to an extra sequence of folding polynomials $P_{\sqrt{2}}, P_{2 \sqrt{2}}, \ldots$ defined by

$$
P_{k \sqrt{2}}\left(h\left[\begin{array}{l}
v_{1} \\
v_{2} \\
v_{3} \\
v_{4}
\end{array}\right]\right)=h\left[\begin{array}{l}
k v_{1}+k v_{2} \\
k v_{2}-k v_{1} \\
k v_{3}+k v_{4} \\
k v_{4}-k v_{3}
\end{array}\right]
$$

and this extra sequence also satisfies the composition property. 


\section{REFERENCES}

1. N. Bourbaki, Groupes et algèbres de Lie, Chapitres IV, V, VI, Hermann, Paris, 1968.

2. H. S. M. Coxeter, Regular polytopes (3rd ed.), Dover, New York, 1973.

3. N. Dunford and J. T. Schwartz, Linear operators, Part I, Interscience, New York, 1958.

4. C. Dunkl, Orthogonal polynomials on the sphere with octahedral symmetry, Trans. Amer. Math. Soc. 282 (1984), 555-575.

5. R. Eier and R. Lidl, A class of orthogonal polynomials in $k$ variables, Math. Ann. 260 (1982), 93-99.

6. H. Hiller, Geometry of Coxeter groups, Pitman, Boston, Mass., 1982.

7. T. Koornwinder, Two-variable analogues of the classical orthogonal polynomials, Theory and Application of Special Functions, R. Askey (ed.), Academic Press, New York, 1975.

8. - Orthogonal polynomials in two variables which are eigenfunctions of two algebraically independent partial differential operators. I-IV, Nederl. Akad. Wetensch. Proc. Ser. A 77 (Indag. Math.) 36 (1974), 48-66 and 357-381.

9. I. G. Macdonald, Symmetric functions and Hall polynomials, Clarendon Press, Oxford, 1979.

10. L. Mittag and M. J. Stephen, Yang-Lee zeros of the Potts model, J. Statist. Phys. 35 (1984), 303-320.

11. T. J. Rivlin, The Chebyshev polynomials, Wiley, New York, 1974.

12. H. Samelson, Notes on Lie algebras, Van Nostrand Reinhold, New York, 1969.

13. L. Schwartz, Mathematics for the physical sciences, Hermann, Paris, 1966.

United States Naval ACademy, AnNapolis, Maryland 21402 